\title{
Reappraising the Diagnostic Accuracy of Post-Treatment Whole-Body Scans for Differentiated Thyroid Carcinoma
}

Authors

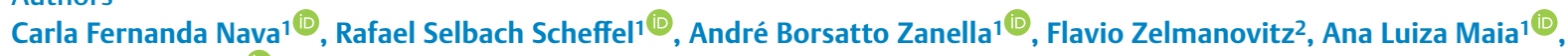
Jose Miguel Dora1'

Affiliations

1 Thyroid Unit, Endocrine Division, Hospital de Clínicas de Porto Alegre, Porto Alegre, Rio Grande do Sul, Brazil

2 Nuclear Medicine, Hospital de Clínicas de Porto Alegre, Porto Alegre, Rio Grande do Sul, Brazil

Key words

differentiated thyroid carcinoma, whole-body scan, radioiodine, prognosis, false-positive

received 01.04 .2020

accepted after revision $\quad 01.07 .2020$

published online $\quad 04.08 .2020$

Bibliography

Horm Metab Res 2020; 52: 834-840

DOI $10.1055 / a-1212-8594$

ISSN 0018-5043

(c) 2020. Thieme. All rights reserved.

Georg Thieme Verlag KG, Rüdigerstraße 14,

70469 Stuttgart, Germany

Correspondence

Jose Miguel Dora MD, PhD

Thyroid Unit

Hospital de Clínicas de Porto Alegre

Rua Ramiro Barcelos 2350, 90035-003

Porto Alegre, RS

Brazil

Tel.: + 55513359 7858, Fax: + 555133325188

jdora@hcpa.edu.br

Ana Luiza Maia MD, PhD

Thyroid Unit

Hospital de Clínicas de Porto Alegre

Rua Ramiro Barcelos 2350, 90035-003

Porto Alegre, RS

Brazil

Tel.: + 55513331 0207, Fax: + 555133325188

almaia@ufrgs.br

\section{ABSTRACT}

Initial treatment for differentiated thyroid carcinoma (DTC) often consists of surgery and the administration of radioiodine. In this context, post-treatment Whole-Body Scans (ptWBS) are currently recommended, but data on its diagnostic accuracy are rare. The aim of the study was to evaluate the performance of ptWBS for distant metastasis in DTC patients. We included DTC patients who received radioiodine and underwent ptWBS between 2009-2015. The medical data were independently reviewed by two specialists to evaluate the concordance of positive distant ptWBS uptake and distant metastasis documented by imaging exams (gold standard). We studied 268 DTC patients. The mean age was $46 \pm 16$ years ( $82 \%$ women), and papillary thyroid carcinoma was diagnosed in $87 \%$ of the patients. The median tumor size was $2.7 \mathrm{~cm}, 40 \%$ had lymph node involvement, and $11 \%$ had distant metastasis. Twenty-eight patients ( $10 \%$ ) had distant ptWBS uptake, and nine of them ( $32 \%$ ) were false-positives. In addition, nine false-negative ptWBS uptakes were identified. The overall performance of ptWBS showed $68 \%$ sensitivity and $96 \%$ specificity with significantly different performance according to the American Thyroid Association (ATA) risk groups. While the ptWBS performance for ATA low-intermediate-risk showed $29 \%$ sensitivity, $97 \%$ specificity, and Kappa of 0.19 , the ATA high-risk group ptWBS displayed high sensitivity ( $82 \%)$, specificity (100\%), and good agreement (Kappa 0.74). ptWBS is useful for a subgroup of ATA high-risk DTC patients. The overall poor performance of ptWBS suggests that it should be reconsidered for routine use in ATA at low to intermediate risk: the exam has little value to this subgroup. 


\section{Introduction}

Differentiated thyroid carcinoma (DTC) comprising papillary (PTC) and follicular carcinoma (FTC) accounts for the majority of all thyroid malignancies [1]. The American Thyroid Association (ATA) Management Guidelines for Adult Patients with DTC [2] recommends initial treatment with surgery and, adjuvant therapies are defined according to risk evaluation as well as the frequency and intensity of follow-up. Radioiodine (RAI) is recommended as adjuvant DTC therapy for remnant thyroid ablation or therapy for persistent and/or metastatic disease [2-4].

Post-treatment Whole-Body Scans (ptWBS) are a mandatory test within the context of patients receiving RAI. The goals of the ptWBS are to identify iodine avidity for structural disease, and to refine disease staging by identifying patients with previously unknown distant metastasis [2]. Prior studies have suggested that about $8 \%$ of DTC cases are reclassified via ptWBS exams as having distant metastasis [5]. Of note, ptWBS seems to be more sensitive to detecting new lesions versus diagnostic Whole-Body Scans (DxWBS) [6]. Data on sensitivity and specificity of the ptWBS exams are scarce and estimated at 65 and $55 \%$, respectively, with a diagnostic accuracy for distant metastasis of $63 \%$ [7].

ptWBS is subject to diagnostic and interpretation problems like other exams [8-13] but is especially plagued by a high false-positive rate. In low-risk DTC populations, the limited detection of distant metastasis restricts its diagnostic utility [14-16]. Remarkably, Garger (2016) reported $73 \%$ of false-positive distant uptakes [17]. False-positive ptWBS is a potential source of unintended psychological stress and wasteful financial health care expenditures.

Any area of the body that concentrates RAI may potentially be interpreted as a region of thyroid cancer metastasis but functional expression of sodium/iodine symporter (NIS) in non-thyroid tissues, iodine retention in body fluids (i. e., cysts, bronchiectasis), technical contamination, infections, and inflammatory processes may exhibit the same pattern of uptake and can cause false-positive findings on ptWBS $[13-15,17,18]$.

The ptWBS also can yield false-negative results. A negative ptWBS may misleadingly suggest the absence of metastatic DTC. In those patients with documented distant metastasis, the negative ptWBS uptake could indicate that an incorrect preparation for RAI administration occurred (inadequate TSH levels, iodine contamination due to the absence of low iodine diet, etc.) or that the tumor underwent a dedifferentiation process [19-21].

The current DTC risk assessment strategies have incorporated clinical and pathological criteria, along with ultrasensitive thyroglobulin $(\mathrm{Tg})$, a marker with high sensitivity and specificity for detection of persistent DTC $[22,23]$. In this context, there is uncertainty regarding the contemporaneous diagnostic usefulness of ptWBS as an adjunct tool for DTC staging.

Thus, the aim of this study was to evaluate the diagnostic value of ptWBS in a cohort of DTC patients in a tertiary, university-based hospital.

\section{Patients and Methods}

\section{Patients and study design}

The subjects were from a cohort of DTC patients from the Thyroid Outpatient Clinic of the Thyroid Unit, Endocrine Division of the Hospital de Clínicas de Porto Alegre (HCPA), a tertiary care, university teaching hospital in southern Brazil. We enrolled all consecutive patients from 2009 to 2015 with a histological diagnosis of DTC who received the first dose of $\mathrm{RAI}$ and had a ptWBS image available. All subjects have given written informed consent.

\section{Ethical approval}

The study was approved by our institution's ethics committee (Ethics and Research Committee of Hospital de Clinicas de Porto Alegre - CAAE 68434617.5.0000.5327/GPPG 17-0482). All methods were performed in accordance with the relevant guidelines and regulations for research involving human database.

\section{Treatment protocol and follow-up}

The DTC treatment protocol consisted of total thyroidectomy followed sometime by administration of RAl as indicated as well as the use of suppressive levothyroxine therapy according to current guidelines [24, 25]. Decisions regarding cervical lymph node dissection were at the discretion of the surgical team. The iodine administration protocol used RAI activities prescribed at the attending physician's discretion. RAl was administered in a stimulated thyrotropin (TSH) condition of endogenous hypothyroidism (TSH > $30 \mathrm{mUI} / \mathrm{l}$ ) after withdrawing levothyroxine (at least 3-4 weeks) and assuming a low iodine diet for 2 weeks. Stimulated postoperative thyroglobulin (sPOTg) was measured at this time. Serum levels of anti-thyroglobulin antibody $(\mathrm{TgAb})$ were measured in the same blood sample, and patients with positive results were excluded from all analyses of sPOTg.

In the first evaluation, the following data were recorded for each patient: demographics, tumor characteristics (e. g., histological features, extension, and lymph node involvement) and treatment (e. g., surgery, RAI, and other interventions). Each patient was classified using the $8^{\text {th }}$ edition of the TNM/AJCC (TNM8) staging system (I, II, III, or IV) [26, 27]. Clinically negative N0 status was determined by clinical examination of the neck or preoperative and postoperative neck ultrasound (US) imaging. Alternatively, we used macroscopic examination during surgery and pathological study of patients with lymph node resection.

The risk of persistent/recurrent disease was assessed based on the proposed risk stratification system by the ATA 2009 risk guideline [28] with patients classified into three risk groups: low (intrathyroidal tumors without any intermediate-high-risk features), intermediate (tumors with microscopic invasion of perithyroidal tissues or cervical lymph node metastasis or tumor with aggressive histology or vascular invasion), and high (tumors with macroscopic tumor invasion, incomplete tumor resection, distant metastasis, and thyroglobulinemia out of proportion to what is seen on the ptWBS).

\section{Post-treatment whole-body scan}

Planar I-131 ptWBS was performed 7 to 10 days after RAI administration in both anterior and posterior projections using a dual- 
detector gamma camera (Infinia, GE Healthcare, Chicago, IL, USA) with high-energy, parallel-hole collimators. Continuous acquisition mode was used at a table speed of $6 \mathrm{~cm} / \mathrm{min}, 1024 \times 256$ matrix, and automated body contour detection applied. The photopeak was centered at $364 \mathrm{keV}$ with a $\pm 10 \%$ window. Additional spot views were acquired at $5 \mathrm{~min} / \mathrm{view}$ with a $256 \times 256$ matrix in the case of unexpected iodine uptake and accumulations suspected to be contamination (scanned after removing the contamination or the stained clothes).

\section{Distant metastasis}

All cases with distant uptake on ptWBS were reviewed by two independent physicians (CFN and JMD). The gold standard for the presence of distant metastasis was an evaluation that considered imaging tests (CT, bone scintigraphy) at the ptWBS uptake sites. Those with distant uptake on ptWBS and imaging tests confirming the presence of distant metastasis in that same topography were classified as true-positives. If the distant uptake on ptWBS was not confirmed by imaging exams, then it was classified as a false-positive uptake.

Furthermore, all patients with a sPOTg $>10 \mathrm{ng} / \mathrm{ml}$ were screened for the presence of distant metastasis by imaging (neck US, CT, and/or bone scintigraphy). Cases with no distant uptake on ptWBS but with proven distant metastasis by other imaging tests were considered false-negatives.

\section{Laboratory analysis}

Serum Tg measurements were conducted using immunoradiometric assays (from 2000 to 2002 via radioimmunoassay; 2002 to 2010 via electrochemiluminescence; and 2010 to the present via chemiluminescence). These assays had a functional sensitivity of $0.2 \mathrm{ng} / \mathrm{ml}$. The TgAb were measured using the passive agglutination method from 2000 to 2010 and by chemiluminescence from 2010 until the present. TSH levels were measured via a chemiluminescence assay from 2000 to 2006 (Immulite 2000 Siemens, Munich, Germany), electrochemiluminescence from 2006 to 2010 (Modular E Roche, Basel, Switzerland), chemiluminescence assay from 2010 to 2014 (Centaur XP Siemens, Munich, Germany), and electrochemiluminescence from 2014 to the present (Cobas E602 ROCHE, Basel, Switzerland). The necessary procedures for standardization and validation were performed after each new assay had been implemented. These tests were all conducted in the HCPA central laboratory.

\section{Statistical analysis}

The clinical and laboratory data are reported as the mean \pm standard deviation (SD) values or as the median and percentiles 25 and 75 (P25-75) for continuous variables or as absolute numbers and percentages for categorical variables. Comparative analyses of frequencies were performed using the Pearson Chi-Square or Fisher's Exact Test as appropriate. These analyses were performed using the Statistical Package for Social Science Professional software version 20.0 (IBM Corp., Armonk, NY, USA).

Sensitivity and specificity of ptWBS were calculated for the overall population sample and specific subgroups. Agreement of ptWBS with the gold standard (clinical and image classification) was accessed using Cohen's Kappa coefficient for two-level mutually exclusive categories using VassarStats. Comparisons of predictive value for ptWBS diagnostic performance between different DTC populations were calculated using the WinPepi version 11.65 with the Weighted Generalized Score test of Kosinski.

Youden's index was calculated using sensitivity and specificity for comparisons of the performance of the ptWBS between two different populations. The Youden index is a measure of test performance and is calculated based on the sensitivity and specificity of the test for a given population (Youden index = sensitivity + specificity-1). Its value ranges from 0 through 1 ; zero means that the diagnostic test gives the same proportion of positive results for groups with and without the disease (i. e., the test is useless) and a value of 1 is when the test is considered perfect (i.e., there are no false-positives or false-negatives) [29]. All tests were two-tailed, and $\mathrm{p}<0.05$ was considered statistically significant.

\section{Results}

\section{Clinical characteristics}

We evaluated 268 patients with clinical and oncological features in - Table 1. The mean age at diagnosis was $46 \pm 16$ years; 220 ( $82 \%$ ) were women, and PTC was diagnosed in 234 (87\%) patients. The median tumor size was $2.7 \mathrm{~cm}$ (P25-75 1.3-3.5 cm). Of these, 107 (40\%) had lymph node metastasis, and 29 (11\%) had distant metastasis. Staging indicated $212(80 \%)$ as stage I, 41 (15\%) as stage II, 2 (1\%) as stage III, and $10(4 \%)$ as stage IV. All patients

\begin{tabular}{l|l|}
$\begin{array}{l}\text { Table } 1 \text { Characteristics of } 268 \text { patients with differentiated } \\
\text { carcinoma. }\end{array}$ & $46 \pm 16$ \\
\hline Age at diagnosis (years) & $220(82)$ \\
\hline Female sex - n (\%) & \\
\hline Histology - n (\%) & $234(87)$ \\
\hline Papillary & $34(13)$ \\
\hline Follicular & $2.7(1.3-3.5)$ \\
\hline Tumor size (cm) & $107(40)$ \\
\hline Lymph node metastasis (N1) - n (\%) & $29(11)$ \\
\hline Distant metastasis - n (\%) & \\
\hline TNM8 - n (\%) & $212(80)$ \\
\hline I & $41(15)$ \\
\hline II & $2(1)$ \\
\hline III & $10(4)$ \\
\hline IV & $106(40)$ \\
\hline ATA Risk - n (\%) & $127(48)$ \\
\hline Low & $32(12)$ \\
\hline Intermediate & $96 \pm 34$ \\
\hline High & \\
\hline Radioactive iodine activity (mCi) & \\
\hline
\end{tabular}

Data are expressed as the mean \pm SD, median (percentiles 2575 ) or frequencies. TNM8: $8^{\text {th }}$ edition of the tumor, node, metastasis stage; ATA risk: American Thyroid Association risk. 


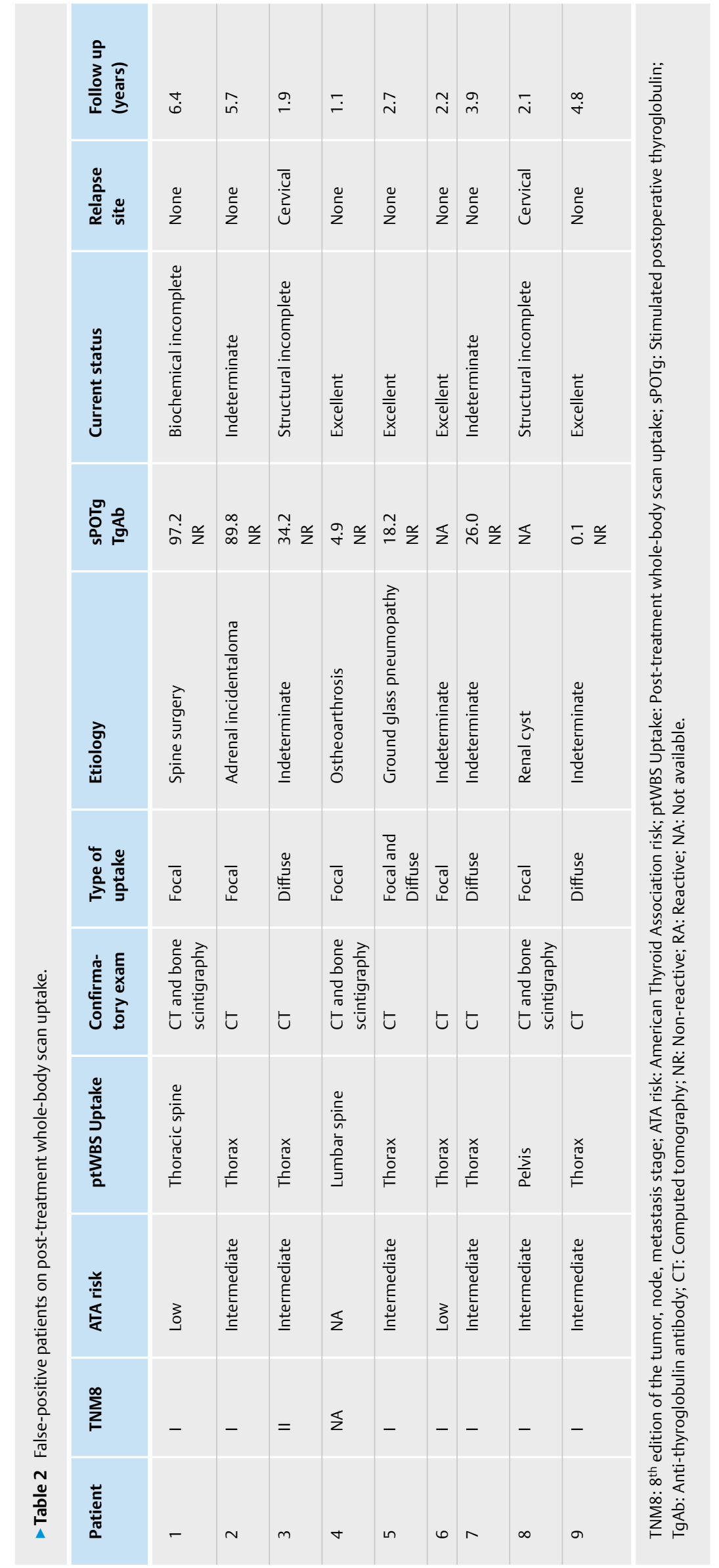


underwent total thyroidectomy and received RAI therapy. The median administered RAl activity was $96 \pm 34 \mathrm{mCi}$.

\section{Post-treatment Whole-Body Scan performance}

Two-hundred and forty ( $90 \%$ ) patients showed no uptake outside the thyroid bed, and $28(10 \%)$ patients displayed distant uptake on ptWBS. Of the 28 patients with distant uptake at ptWBS, distant disease was confirmed in 19 (68\%, true-positives), and nine (32\%) were false-positives. The false-positive uptakes on ptWBS were as follows: six on the thorax, two on the spine, and one on the pelvis. Further studies clarified the false-positive uptakes and revealed two cases of osteoarthrosis (inflammatory), one renal cyst, and one adrenal incidentaloma ( $\vee$ Table 2 ). Of note, no cause could be defined for five false-positive ptWBS uptakes (indeterminate etiology).

Of the 240 patients with no uptake outside the thyroid bed, nine (4\%) had distant metastasis and were considered false-negative on ptWBS. The patterns of metastasis of the false-negative uptake included five patients with pulmonary, two with bone, and two with multiple metastasis sites.

The overall sensitivity of ptWBS for detecting distant metastasis was $68 \%$ with a specificity of $96 \%$. The Youden index was 0.64 and Kappa agreement with the gold standard of 0.66 .

\section{ptWBS Performance According to ATA Risk}

We next explored the ptWBS performance according with the ATA risk. Of the 268 DTC patients, 106 (40\%) were classified as ATA lowrisk, 127 (48\%) ATA intermediate-risk, and 32 (12\%) ATA high-risk. Three patients were excluded due to a lack of information for ATA risk classification

In the low-risk group, only 3 patients (3\%) showed distant ptWBS uptake outside the thyroid bed. Of these, two were considered false-positives and one was a true-positive (29years-old, man, pT2 Nx Mx, with diffuse and focal ptWBS lung uptake and thorax CT micronodules; who was classified as biochemical incomplete response after three years of follow up). No false-negative ptWBS occurred in this group. The sensitivity and specificity of ptWBS for distant metastasis were 33 and $98 \%$, respectively. The performance of ptWBS for ATA low-risk patients had a Youden index 0.31 and a Kappa of 0.27 .

The group classified as ATA intermediate-risk included 121 patients (95\%) with no ptWBS uptake outside thyroid bed, and 6 (5\%) with distant uptake. Of these 6, 5 were considered false-positives, and one was a true-positive ( 25 years-old, woman, pT2 N1b Mx; with cervical, mediastinal and lung (focal and diffuse) ptWBS uptake. Cervical and thorax CT were unremarkable, stimulated Tg $66.6 \mathrm{ng} / \mathrm{ml}$. After three years of follow up the patients was classified as biochemical incomplete response). False-negative ptWBS occurred in three (2\%) intermediate-risk patients rendering a ptWBS sensitivity of $25 \%$ and a specificity of $96 \%$ for this subgroup. The performance of ptWBS included a Youden index of 0.21 and a Kappa of 0.19 for ATA intermediate-risk patients.

Among the 32 ATA high-risk patients, 13 (40\%) had distant metastasis ( 3 diagnosed before and 10 after total thyroidectomy but before ptWBS) and 19 (60\%) had locally advanced disease. Of all high-risk patients, 14 (44\%) showed no uptake outside thyroid bed on ptWBS, and 18 (56\%) had distant uptake. All positive ptWBS were considered true-positives. There were $4(13 \%)$ false-negative exams in the negative ptWBS uptakes. These figures resulted in a ptWBS sensitivity

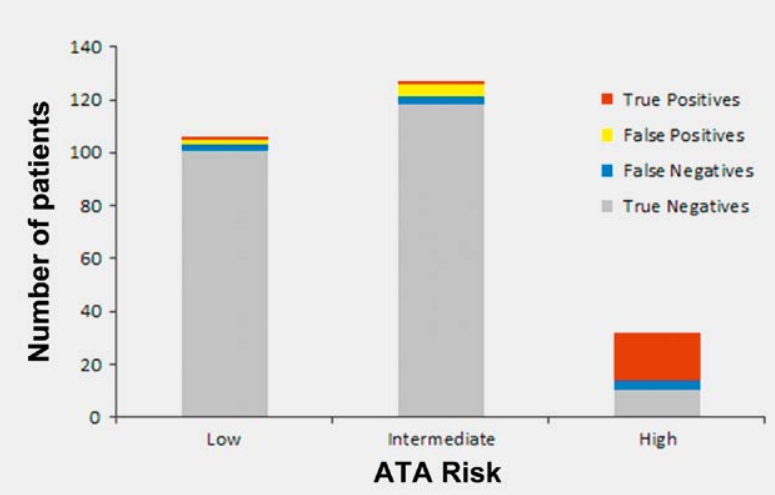

- Fig. 1 Post-treatment Whole-Body Scan uptake, stratified by the American Thyroid Association (ATA) risk.

- Table 3 Performance of post-treatment whole-body scan for diagnosis of distant metastasis between different groups of differentiated thyroid carcinoma.

\begin{tabular}{|c|c|c|c|c|c|c|c|}
\hline & \multirow[t]{2}{*}{ All $(n=268)$} & \multicolumn{3}{|c|}{ American Thyroid Association Risk $(n=265)^{*}$} & \multicolumn{3}{|c|}{$\begin{array}{l}\text { Stimulated Postoperative Thyroglobulin } \\
\qquad(\mathrm{n}=174)^{*}\end{array}$} \\
\hline & & Low $(n=106)$ & $\begin{array}{l}\text { Intermediate } \\
(n=127)\end{array}$ & $\begin{array}{l}\text { High } \\
(\mathrm{n}=32)\end{array}$ & $\begin{array}{l}<1 \mathrm{ng} / \mathrm{ml} \\
(\mathrm{n}=28)\end{array}$ & $\begin{array}{l}1-10 \mathrm{ng} / \mathrm{ml} \\
(\mathrm{n}=71)\end{array}$ & $\begin{array}{l}>10 \mathrm{ng} / \mathrm{ml} \\
(\mathrm{n}=75)\end{array}$ \\
\hline Sensitivity & 0.68 & 0.33 & 0.25 & 0.82 & NA & 0.67 & 0.69 \\
\hline Specificity & 0.96 & 0.98 & 0.96 & 1.00 & 0.96 & 0.99 & 0.92 \\
\hline Youden's index & 0.64 & 0.31 & 0.21 & 0.82 & NA & 0.66 & 0.61 \\
\hline Kappa & 0.66 & 0.27 & 0.19 & 0.74 & 0 & 0.23 & 0.70 \\
\hline
\end{tabular}


and specificity of 82 and $100 \%$, respectively ( performance of ptWBS for ATA high-risk patients showed a Youden's index of 0.82 and a Kappa of 0.74 .

The weighted generalized score was used to compare the diagnostic performance of ptWBS among the different ATA risk DTC populations. For low-intermediate versus high-risk, the Youden index was 0.26 versus $0.82(p=0.003)$, respectively. When we excluded ATA low-risk patients and compared ATA intermediate-risk versus high-risk patients, the Youden index was 0.21 versus 0.82 $(p \leq 0.0001)$, respectively.

\section{ptWBS Performance according to sPOTg}

As an additional analysis, we stratified the DTC cohort according to sPOTg into three categories: $<1.0 \mathrm{ng} / \mathrm{ml}$, between $1-10 \mathrm{ng} / \mathrm{ml}$, and $>10 \mathrm{ng} / \mathrm{ml}$. Here, 174 patients with negative $\mathrm{TgAb}$ values were included (94 were excluded due to a positive $\mathrm{TgAb}$ result). Twenty-eight patients had a sPOTg $<1.0 \mathrm{ng} / \mathrm{ml}, 71$ patients had a sPOTg $1-10 \mathrm{ng} / \mathrm{ml}$, and 75 had a sPOTg $>10 \mathrm{ng} / \mathrm{ml}$.

We compared the performance of ptWBS for patients with sPOTg $\leq 10.0 \mathrm{ng} / \mathrm{ml}$ versus sPOTg $>10 \mathrm{ng} / \mathrm{ml}$ : The sensitivity values were $67 \%$ versus $69 \%$, specificity was $98 \%$ versus $92 \%$, and the Youden index 0.65 versus $0.61(p=0.69)$, respectively. Comparing only patients with sPOTg $1-10 \mathrm{ng} / \mathrm{ml}$ versus sPOTg $>10 \mathrm{ng} / \mathrm{ml}$ the sensitivity was $67 \%$ versus $69 \%$, specificity was $99 \%$ versus $92 \%$, and the Youden index was 0.66 versus $0.61(p=0.95)$ ( $\triangleright$ Table 3$)$.

\section{Discussion}

Management of DTC patients is under continuous review and has changed substantially in recent years. The ptWBS test has pitfalls that limit its performance to the diagnosis of distant disease mainly due to false-positive uptakes in patients with a low pre-test probability of metastasis, jeopardizing the current recommendation for ptWBS in all DTC patients. Indeed, the ptWBS performance for the overall DTC population studied had $68 \%$ sensitivity, $96 \%$ specificity, a Youden index of 0.64 , and a Kappa of 0.66 . We also showed that the ptWBS contribution for disease management varies according to ATA risk categories with poor performance for ATA low and intermediate-risk DTC patients (Youden index of 0.26). In ATA highrisk DTC patients, however, the ptWBS has a good performance to identify those with distant metastasis (Youden index of 0.82 ).

Recent studies have raised questions on the contemporaneous usefulness of ptWBS as an adjunct diagnostic tool for DTC patients. Garger et al. showed that the incidence of ptWBS false-positives is around $73 \%$ with predominance for focal uptake. The etiology of false-positive uptakes cannot be determined in one-third of patients [17, 30]. Accordingly, we analyzed ptWBS after the first activity of RAl and found five false-positive results patterns. The most frequent are diffuse uptake on the thorax. Of note, in 5/9 (56\%) patients we could not determine the etiology of the falsepositive uptake. These findings are concerning because they add undesirable psychological stress and waste healthcare resources.

We further explored the performance of the ptWBS exam according to DTC patient characteristics. When stratifying the DTC population according to the ATA risk, the performance of ptWBS for ATA low-intermediate-risk was poor with a predominance of false-positive uptakes over true-positives. This leads to a higher risk of misdiagnosing patients. Fewer than $1 \%$ of low-intermediate-risk patients could benefit from ptWBS. In addition, only two patients stratified as ATA low to intermediate risk with true-positive ptWBS, met sPOTg values criteria for distant metastasis screening (sPOTg $>10 \mathrm{ng} / \mathrm{ml}$ ). Thus, ptWBS did not bring additional benefit to the follow-up strategy.

We also analyzed the ptWBS performance for the subgroup of ATA intermediate-risk patients, and the performance of the exam was also poor (sensitivity $25 \%$ and specificity $96 \%$ ). These findings agree with recent data published by Agate et al. that showed a low diagnostic value of ptWBS for low and intermediate risk DTC patients [16].

On the other hand, our data illustrates that ptWBS has high sensitivity (82\%), specificity (100\%), and good agreement (Kappa 0.74 ) for ATA high-risk patients. It is useful in the diagnosis of distant metastasis in a substantial portion of this subgroup of patients. These results suggest that if the pre-test probability for distant metastasis is high, then the exam has a positive predictive value of $100 \%$.

The role of sPOTg has been well established in adult DTC populations. Thus, we further stratified the DTC cohort according to sPOTg levels using a cut-off point of $10 \mathrm{ng} / \mathrm{ml}$ as suggested by a systematic review of sPOTg prognostic value [23]. However, the sPOTg cut-off of $10 \mathrm{ng} / \mathrm{ml}$ was not adequate to identify subgroups of DTC patients with better ptWBS performance.

Some limitations of our study should be acknowledged. The relatively low number of high-risk patients raises for caution on definitive conclusions for this subgroup of individuals. Also, the 2015 ATA Guidelines updated the ATA 2009 risk stratification system, incorporating BRAFV600E tumor mutation status and detailed information on the number and size of lymph node metastasis. Whereas the new system may provide an improvement in the risk assessment, it also poses barriers regarding its applicability. Unfortunately, the retrospective design of our study did not allow to incorporate the ATA 2015 risk stratification system, given that we could not obtain all required criteria to consistently classify our patients. Also, we were not able to perform 18-FDG-PET/CT studies since only recently, this technique was available (although still of restricted use) in our center. Another aspect that merits consideration is that we did not use ptWBS associated with SPECT/CT. SPECT/CT improves the diagnostic accuracy of ptWBS [7] and has been a useful tool to clarify localization of ptWBS uptakes. However, in this study we exclusively evaluated the performance of ptWBS considering that the SPECT/CT is not available in many healthcare scenarios, a common place reality for many healthcare facilities in low and middle income countries. Finally, the study was conducted in a single DTC referral center. Thus, it would be of interest to replicate this study in different contemporaneous cohorts.

In summary, our results suggest that we should reconsider the routine use of ptWBS for all DTC patients. It remains useful in ATA high-risk DTC patients.

\section{Author Contributions}

CFN, RSS, ALM, and JMD contributed to the study conception, design, data analysis, interpretation, and manuscript preparation. CFN, RSS, ABZ, FZ, ALM, and JMD were responsible for data collection, data analysis, and manuscript preparation. All authors read and approved the final manuscript version. 


\section{Funding Information}

This work has been made possible by grants from Conselho Nacional de Desenvolvimento Científico e Tecnológico (CNPq), Coordenação de Aperfeiçoamento de Pessoal de Nível Superior (CAPES), Fundo de Incentivo à Pesquisa (FIPE/HCPA), and Programa de Apoio a Núcleos de Excelência (PRONEX)/Fundação de Amparo à Pesquisa do Estado do Rio Grande do Sul (FAPERGS).

\section{Availability of Materials and Data}

All medical records are restricted to maintain patient privacy and avoid unnecessary exposure but can be assessed under request.

\section{Acknowledgements}

We thank Andreia C. F. da Silveira for helping with nuclear medicine and Aline Castello Branco Mancuso for statistical help.

\section{Conflict of Interest}

The authors declare that they have no conflict of interest.

References

[1] Bray F, Ferlay J, Soerjomataram I et al. Global cancer statistics: GLOBOCAN estimates of incidence and mortality worldwide for 36 cancers in 185 countries. CA Cancer J Clin 2018; 68: 394-424

[2] Haugen BR, Alexander EK, Bible KC et al. 2015 American thyroid association management guidelines for adult patients with thyroid nodules and differentiated thyroid cancer: The american thyroid association guidelines task force on thyroid nodules and differentiated thyroid cancer. Thyroid 2016: 1-133

[3] Ahn BC. Sodium iodide symporter for nuclear molecular imaging and gene therapy: From bedside to bench and back. Theranostics 2012; 2: 392-402

[4] Chung JK. Sodium iodide symporter: Its role in nuclear medicine. J Nucl Med 2002; 43: 1188-1200

[5] Pacini F, Agate L, Elisei R et al. Outcome of differentiated thyroid cancer with detectable serum $\mathrm{Tg}$ and negative diagnostic 131 I whole body scan: Comparison of patients treated with high $131 \mathrm{I}$ activities versus untreated patients. J Clin Endocrinol Metab 2001; 86: 4092-4097

[6] Fatourechi V, Hay ID, Mullan BP et al. Are posttherapy radioiodine scans informative and do they influence subsequent therapy of patients with differentiated thyroid cancer? Thyroid 2000; 10: 573-577

[7] Oh JR, Byun BH, Hong SP et al. Comparison of 131 I whole-body imaging, ${ }^{131}$ S SPECT/CT, and ${ }^{18} \mathrm{~F}$-FDG PET/CT in the detection of metastatic thyroid cancer. Eur J Nucl Med Mol Imaging 2011; 38: 1459-1468

[8] Yildirim-Poyraz N, Ozdemir E, Amutkan C et al. False-positive iodine-131 whole body scan due to a benign dermal lesion; Intradermal nevus ((131)I uptake in a benign nevus). Ann Nucl Med 2013; 27: 786-790

[9] Chaussé G, Kader T, Abikhzer $G$ et al. Asthmatic exacerbation as a cause of false-positive whole-body iodine scan in a patient with treated papillary thyroid carcinoma. Clin Nucl Med 2018; 43: 256-257

[10] Ceccarelli C, Pacini F, Lippi F et al. An unusual case of a false-positive iodine-131 whole body scan in a patient with papillary thyroid cancer. Clin Nucl Med 1988; 13: 192-193

[11] Wang R, Zhou K, Fan Q et al. A false-positive I-131 finding of duodenum diverticulum in thyroid cancer evaluation by SPECT/CT: A case report. Medicine (Baltimore) 2018; 97: e9997
[12] Maciejewski A, Czepczyński R, Ruchala M. False-positive radioiodine whole-body scan due to a renal cyst. Endokrynol Po 2018; 69: 736-739

[13] Shapiro B, Rufini V, Jarwan A et al. Artifacts, anatomical and physiological variants, and unrelated diseases that might cause false-positive whole-body 131-I scans in patients with thyroid cancer. Semin Nucl Med 2000; 30: 115-132

[14] Buton L, Morel O, Gault P et al. False-positive iodine-131 whole-body scan findings in patients with differentiated thyroid carcinoma: Report of 11 cases and review of the literature. Ann Endocrinol (Paris) 2013; 74: $221-230$

[15] Hannoush ZC, Palacios JD, Kuker RA et al. False positive findings on I-131 WBS and SPECT/CT in patients with history of thyroid cancer: Case series. Case Rep Endocrinol 2013; 8568347

[16] Agate L, Bianchi F, Brozzi F et al. Less than $2 \%$ of the low- and intermediate-rlsk differentiated thyroid cancers show distant metastases at post-ablation whole-body scan. Eur Thyroid J 2019; 8: 90-95

[17] Garger YB, Winfeld M, Friedman K et al. In thyroidectomized thyroid cancer patients, false-positive I-131 whole body scans are often caused by inflammation rather than thyroid cancer. J Investig Med High Impact Case Rep 2016; 25: 2324709616633715

[18] Oh JR, Ahn BC. False-positive uptake on radioiodine whole-body scintigraphy: physiologic and pathologic variants unrelated to thyroid cancer. Am J Nucl Med Mol Imaging 2012; 2: 362-385

[19] Ma Chao, Kuang Anren, Xie Jiawei et al. Possible explanations for patients with discordant findings of serum thyroglobulin and ${ }^{131}$ | whole-body scanning. J Nucl Med 2005; 46: 1473-1480

[20] Durante C, Puxeddu E, Ferretti E et al. BRAF mutations in papillary thyroid carcinomas inhibit genes involved in iodine metabolism. J Clin Endocrinol Metab 2007; 92: 2840-2843

[21] Chakravarty D, Santos E, Ryder M et al. Small-molecule MAPK inhibitors restore radioiodine incorporation in mouse thyroid cancers with conditional BRAF activation. J Clin Invest 2011; 121: 4700-4711

[22] Baudin E, Do Cao C, Cailleux AF et al. Positive predictive value of serum thyroglobulin levels, measured during the first year of follow-up after thyroid hormone withdrawal, in thyroid cancer patients. J Clin Endocrinol Metab 2003; 88: 1107-1111

[23] Webb RC, Howard RS, Stojadinovic A et al. The utility of serum thyroglobulin measurement at the time of remnant ablation for predicting disease-free status in patients with differentiated thyroid cancer: A meta-analysis involving 3947 patients. J Clin Endocrinol Metab 2012; 97: 2754-2763

[24] Scheffel RS, Zanella AB, Dora JM et al. Timing of radioactive iodine administration does not influence outcomes in patients with differentiated thyroid carcinoma. Thyroid 2016; 26: 1623-1629

[25] Rosario PW, Ward LS, Carvalho GA et al. Thyroid nodules and differentiated thyroid cancer: Update on Brazilian consensus. Arqui Brasil. Endocrinol Metab 2013; 57: 205-232

[26] Tuttle M, Morris LF, Haugen B et al. Thyroid-differentiated and anaplastic carcinoma (chapter 73). AJCC Cancer Staging Manual. $8^{\text {th }}$ ed. Edge MB, Greene SB, Byrd F et al. (eds). New York, NY, USA: Springer International Publishing; 2017: 873-890

[27] Nava CF, Zanella AB, Scheffel RS et al. Impact of the updated TNM staging criteria on prediction of persistent disease in a differentiated thyroid carcinoma cohort. Arch. Endocrinol Metab 2019; 63: 5-11

[28] Cooper DS, Dohertu GM, Haugen BR et al. Revised American Thyroid Association management guidelines for patients with thyroid nodules and differentiated thyroid cancer. Thyroid 2009; 19: 1167-1214

[29] Youden WJ. Index for rating diagnostic tests. Cancer 1950; 3: 32-35

[30] Oral A, Yazici B, Eraslan C et al. Unexpected false-positive I-131 uptake in patients with differentiated thyroid carcinoma. Mol Imaging Radionucl Ther 2018; 9: 99-106 